\title{
1 ICOS-L as a Potential Therapeutic Target for Cancer 2 Immunotherapy
}

Marinelli Oliviero ${ }^{1, *}$, Nabissi Massimo ${ }^{1, * \#}$, Morelli Maria Beatrice ${ }^{1}$, Amantini Consuelo ${ }^{2}$, Torquati Luciana ${ }^{3}$, Santoni Giorgio ${ }^{1}$,

1) School of Pharmacy, University of Camerino, Camerino (MC), Italy

2) School of Bioscience and Veterinary Medicine, University of Camerino, Camerino (MC), Italy

3) Exeter University Medicine School, University of Exeter, Exeter, United Kingdom

4) School of Human Movement and Nutrition Sciences, University of Queensland, Queensland, Australia

\author{
* Equally contribute \\ \#Corresponding author: Dr. Massimo Nabissi, School of Pharmacy, Dept. Experimental Medicine, via Madonna \\ delle Carceri 9, 62032 Camerino (MC), Italy. e-mail: massimo.nabissi@unicam.it, phone:+390737403306, \\ fax:+390737403325
}

\section{Graphical Abstract}

Abstract

Background: The co-stimulatory B7 family members are cell-surface protein ligands, binding to receptors on lymphocytes to regulate immune responses. One of them is the inducible co-stimulatory molecule ligand (ICOS-L). This is expressed on professional antigen-presenting cells (APCs), including B cells, macrophages, and dendritic cells (DCs), but it can also be expressed by endothelial cells, lung epithelium and in tumour microenvironment cells. ICOS-L is important for memory and effector $\mathrm{T}$ cells during the specific humoral immune responses, but its role in cancer is not yet understood. Objective: To discuss the role of ICOS/ICOS-L in cancer, given importance of identifying selective targets for cancer treatment and knowing the mechanism of immune evasion by tumour

Main findings: ICOS/ICOS-L signal has opposite effects on the T-cell response. ICOS-L is activated in several types of cancers to maintain immunosuppressive $\mathrm{CD}^{+} \mathrm{T}$ cell subsets, such as regulatory $\mathrm{T}$ cells (Rs). ICOS-L over-expression is associated with tumour progression and poor overall survival. In colon cancer, activation of this co-stimulatory signal is associated with improved survival suggesting a dualistic effect of the ICOS/ICOs-L signal pathway. Interestingly, following anti-cancer vaccine or anti-CTLA- 4 treatment, $\mathrm{ICOS}^{+} \mathrm{T}$ cells increased significantly in both the $\mathrm{CD}^{+}$and $\mathrm{CD}^{+}$population and the ratio Teff/Treg increased in tumour microenvironment. Thus suggesting a potential role of ICOS/ICOs-L in improving effectiveness of cancer therapy.

Conclusion: ICOS/ICOS-L signal pathway has the potential to improve cancer treatment. However, future studies in other models are needed to understand whether inhibition of ICOS expression or the blockage of its co-stimulation could be a potential therapeutic target or adjuvant treatment for immunotherapy.

Keywords: ICOS-L, ICOS, CD275, CD278, B7, Tregs, cancer 


\subsection{ICOS-L, a member of co-stimulatory B7 family.}

The co-stimulatory B7 family members are cell-surface protein ligands, binding to receptors expressed on lymphocytes and involved in regulating immune responses. This system not only provides positive signals to stimulate T-cell activation, but also it delivers negative signals to inhibit T-cell responses. The inducible co-stimulatory molecule ligand (ICOS-L), also known as B7RP-1, B7-H2, LICOS, GL50, B7h and CD275, is a member of B7 family. It is expressed on professional antigen-presenting cells (APCs), including B cells, macrophages and dendritic cells (DCs), but also in certain endothelial cells and lung epithelium [1-3]. Despite limited evidence, ICOS-L is known to act as a co-stimulatory signal for T-cell proliferation and cytokines secretion and to induce B-cell proliferation and differentiation into plasma cells. ICOS-L could also play an important role in mediating local tissue responses to inflammatory conditions and in modulating the secondary immune response by co-stimulating memory T-cell function [2, 3].

\subsubsection{ICOS-L: gene, transcripts and proteins.}

The gene encoding the inducible co-stimulator ligand (ICOS-L), is located in the 21q22.3, is 24,477 bases in length and contains 10 exons [4]. At transcriptional level, four transcript variants (Var) have been cloned. Vara (the

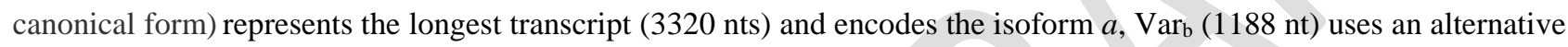
3' terminal exon and it thus differs in the 3' coding region and 3' UTR, compared to $\operatorname{Var}_{\mathrm{a}}$. $\operatorname{Var}_{\mathrm{c}}$ (2969 nt) lacks an alternate in-frame exon in the 5' coding region, compared to $\operatorname{Var}_{\mathrm{a}}$, resulting in an isoform $c$ that is shorter than isoform a. $\operatorname{Var}_{\mathrm{d}}$ (3168 nt) uses an alternate splice site in the 5' region, resulting in translation initiation at a downstream in-frame start codon, compared to $\operatorname{Var}_{\mathrm{a}}$. The encoded isoform $d$ is shorter at the N-terminus, compared to isoform $a$ (Fig.1, Supplementary 1). Isoform $a$ is widely expressed (brain, heart, kidney, liver, lung, pancreas, placenta, skeletal muscle, bone marrow, colon, ovary, prostate, testis, lymph nodes, leukocytes, spleen, thymus and tonsil), while isoform $b$ is detected only in lymph nodes, leukocytes, spleen and it is expressed on activated monocytes and dendritic cells [5]. Tissue and organ expression of $\operatorname{Var}_{\mathrm{c}}$ and $\mathrm{Var}_{\mathrm{d}}$ are yet to be studied.

\subsection{Immunological role of ICOS-L}

ICOS-L specifically binds the T-cell inducible co-stimulatory molecule (ICOS), also named CD278 [3], which is expressed at high levels in germinal center T cells or TFH (follicular helper) cells. Activation of ICOS receptor induces recruitment of class IA phosphatidylinositol 3-kinase (PI3K), a signalling molecule that leads to the production of membrane-bound phosphatidylinositol 3,4,5-trisphosphate (PIP3). This culminates in the activation of Akt, a kinase that promotes cellular proliferation and survival. ICOS activation also recruits the p50 $\alpha$ and p85 $\alpha$ regulatory subunits and $\mathrm{p} 110 \delta$ catalytic subunit of PI3K. In vivo and in vitro experiments indicate that ICOS/ICOS-L pathway activation contributes to the production of cytokines, such as IL-5, IL-4,IL-10 and IL-13. ICOS/ICOS-L co-stimulatory signal is important for memory and $\mathrm{T}$ helper cell effector functions. This is particularly important for $\mathrm{T}$ helper-2 differentiation and antibodies response during the specific humoral immune responses against pathogens such as bacteria, parasites, and viruses [1,2]. Moreover, ICOS-L seem to alternatively promote or repress T helper-1 responses under different infection conditions and these divergent phenotypes may in part be explained by ICOS-dependent regulatory T cells (Tregs) induction (Fig. 2). Thus, the ability of ICOS-L to influence Tregs induction points to a complex role in regulating CD4 ${ }^{+}$ $\mathrm{T}$ cell differentiation, and suggests that ICOS-L signalling may be mutually important in preventing immune-mediated pathology, as well as inducing pro-inflammatory $\mathrm{CD}^{+}{ }^{+} \mathrm{T}$ cells. 


\subsection{ICOS- $L$ in tumour infiltrate lymphocytes}

Malignant cells may adopt several mechanisms to interfere with the effector immune response or with regulatory cells in tumour microenvironment, in order to escape from immune surveillance. Regulatory T cells (Tregs) constitute $5 \%$ to $10 \%$ of all peripheral $\mathrm{CD}^{+} \mathrm{T}$ cells and play an important role in maintaining tolerance to both auto-immune and cancer cells. Starting from the identification of Forkhead box protein 3 (Foxp3) as a critical transcriptional factor for Tregs, Foxp $3^{+} \mathrm{CD}^{+} \mathrm{T}$ cells have been regarded as Tregs with immunosuppressive functions and divided in three subpopulations: effector Tregs (eTregs), naive Tregs, and non-Tregs [6]. Tregs, like other T cells, respond to TCR stimulation. They also express co-stimulatory receptors, such as CTLA-4, PD-1, ICOS and CD28 that further promote their activation, proliferation and survival(ref). A large body of evidence confirms that activation of ICOS/ICOS-L pathway is involved in maintenance of this subtype of $\mathrm{T}$ cells in tumour microenvironment, which usually is associated with a poor prognosis of patient [6].

\subsubsection{ICOS-L in cancer}

ICOS/ICOS-L pathway was reportedly activated in melanoma [7], myeloma [8,9], breast [10], ovarian [11], gastric [6], liver [12] and colorectal cancers [13]. Furthermore, ICOS-L was also found in classical Hodking lymphoma [14], B lymphoma [2], leukemia [15], glioblastoma [16] and rhabdomyosarcoma [17], but its role in these cancers is yet to be completely elucidated. In tumour microenvironment, Treg cells contribute to tumour growth by inhibiting tumourspecific immunity through not yet characterized mechanisms. It is hypothesised that this mechanism may involve FASL, CD39/Adenosine, perforins, CTLA-4, or PD-1 and the generation of high levels of IL-10, which mediate a suppressive capability, especially against dendritic cell functions. Finally, it was demonstrated that ICOS/ICOS-L pathway could modulate the efficacy of therapy in patients with prostate cancer [18], pancreatic cancer [19], bladder cancer [20], multiple myeloma [21] and melanoma [22,23].

\subsubsection{ICOS-L in melanoma}

Melanoma tumour biopsies analysis showed an estimated $25 \%$ of the $\mathrm{CD}^{+} \mathrm{T}$ cells found were Foxp3 $3^{+}$, more than 3 times the abundance found in the peripheral blood of the same patients (7\%).This indicates that ICOS-L expressed by melanoma cells could co-stimulate Tregs to induce high levels of Foxp3, CD25, ICOS and also production of IL-10. This evidence suggest that tumour cells may act themselves as direct APC, since they can express HLA class II and provide self-antigen presentation and co-stimulation through ICOS-L, allowing a tumour self-tolerance [7].

\subsubsection{ICOS-L in haematological tumors}

In several haematological malignancies, ICOS-L was expressed in cancer cells. Malignant Hodgkin Reed Sternberg cells derived from a germinal center B cells over-expressed different surface molecules, including CD70, CD80/CD86, CD30, CD40, OX40-L/CD252, and ICOS-L. Their respective receptors were often present on typical Hocking lymphoma microenvironment immune cells and was considered to be up-regulated on activated T Helper cells (ref). Evidence suggests that malignant tumours were capable of sequestering a substantial number of activated functional $\mathrm{T}$ cells, contributing to a profound systemic immune deficit in advanced disease [14]. Additionally, it was shown that leukemic cells, obtained from patients with acute myeloid leukemia, express ICOS-L and this it was associated with a poor prognosis(ref). Thus suggesting that expression of ICOS-L in leukemic cells might contribute to their proliferation by helping them to evade antitumor immune responses [15]. Although ICOS-L expression has been evaluated on B lymphoma tissues, its biological functions in regulating anticancer immune response is not completely understood [2]. 
Immunohistochemical staining showed that ICOS-L was expressed in thyroid B-cell lymphoma but not in thyroid adenoma tissue or healthy thyroid tissue, suggesting that ICOS-L molecules may be involved in the development of malignant B lymphoma [2]. In an in vitro model of myeloma, tumour cells express ICOS-L [8,9] which directly induces not only Tregs expansion but also Treg cells generation in a contact-dependent manner and in absence of APC cells. The induction of Treg cells mediated by cell-to-cell contact with the ICOS/ICOS-L system supports the hypothesis that this pathway may play a central role in the immune suppression response [8].

\subsubsection{ICOS-L in ovarian and breast cancer}

While previously described evidence indicated ICOS-L expression in tumour cells, recent investigations in ovarian cancer and breast cancer are in contrast with this theory. In ovarian and breast cancer, ICOS-L was not expressed in cancer cells indicating that Tregs expansion was activated by other cells expressing ICOS [10,11]. In ovarian cancer many Foxp $3^{+}$Treg cells were found to infiltrate the tumour microenvironment, a phenomenon that strongly predicts disease progression. These Tregs constitutively expressed ICOS and were frequently detected in the tumour of patients with epithelial ovarian cancer. Survival, proliferation and functions of ICOS ${ }^{+} \mathrm{Foxp}^{+}$Treg cells was strictly dependent to the presence of tumour-infiltrating plasmacytoid dendritic cells (pDCs) [11]. Human DCs consist of two subsets, myeloid dendritic cells (mDCs) and plasmacytoid dendritic cells (pDCs). The first derive from TLR2-6, 8 and induce T helper 1 effector cells, while pDCs mature from TLR7 and 9 and have been reported to exist in most human solid tumours. pDCs are associated with induction and maintenance of immunosuppressive conditions by the secretion of indoleamine 2,3dioxygenase, IL-3, expression of ICOS-L, CD40-L and through Treg cells activation. There is substantial evidence suggesting that pDCs have a specialized role in the induction of peripheral tolerance by inducing IL-10-production through Tregs-ICOS-L/ICOS signalling [6,24]. In summary, in epithelial ovarian cancer the ICOS/ICOS-L pathway activation involveed a tumor-infiltrating pDCs that express high levels of ICOS-L, while cancer cells did not express significant levels of this protein. This evidence supports the hypothesis that tumor-infiltrating pDCs were directly involved in creating an immunosuppressive tumour microenvironment through the expansion of the ICOS ${ }^{+} \mathrm{Foxp}^{+} \mathrm{Treg}^{-}$ cell subset [11].

Likewise, infiltration of pDS indirectly induced by ICOS was associated with poor prognosis of breast cancer patients [10]. While ICOS-L was not expressed in neither primary breast tumour cells or in breast tumour cell lines, ICOS engagement was described as a major contributing pathway to ICOS ${ }^{+}$Tregs local accumulation. Tumour-associated ICOS ${ }^{+}$Tregs directly interacted with pDCs in situ, as confirmed by immunohistochemistry on primary breast tumour frozen sections. Upstream of this cascade, CCL22 was produced by tumor cells to recruit CCR4+Treg cells from the bloodstream to breast tumour microenvironment. This resulted in CCR4+ Treg cells local expansion through ICOS/ICOSL interaction with pDC in breast tumour environment [10].

\subsubsection{ICOS- $\mathrm{L}$ in gastric and liver cancers}

In gastric cancer, a high number of ICOS+ cells were found in gastric tissue of late-stage gastric cancer patients. In this patients, the expression level of ICOS-L was high in pDCs while high ICOS expression in Foxp $3^{+}$infiltrating T lymphocyte was associated with a poor prognosis. Immunohistochemistry results showed a coexistence of ICOS ${ }^{+}$Foxp $3^{+}$ cells and pDCs in gastric cancer tissues suggesting some functional relationship between these cells, as seen in other tumour types [6]. Additionally, this ICOS/ICOS-L pathway was not only involved in regulating the Treg cell subset but also other types of inhibitory $\mathrm{T}$ cells potentially involved in local immunosuppression. Among these, T regulatory type 1 cells (Treg1), initially considered important in promoting and maintaining tolerance in autoimmunity, allergy and 
transplantation, also demonstrated to be involved in promoting tumour escape from immune surveillance [12]. In patients with primary and secondary liver cancer, tumour infiltrating Treg1 cells contributed to local immune suppression via ICOS/ICOS- L signalling and ICOS-L+pDCs stimulated IL-10 production in the tumour microenvironment [12].

\subsubsection{ICOS-L in others solid cancers.}

ICOS-L was found expressed in human glioma cells, both in vitro and in vivo but not in normal central nervous system tissues adjacent to the neoplastic cells. Whether ICOS-L plays a role in supporting theses malignancy is yet to be investigated [16]. In rhabdomyosarcoma (RMS), the most common paediatric soft tissue malignancy, ICOS-L was expressed in FLOH1, RH41, RD6, and TE671 RMS cell lines and its expression levels marginally increased in presence of TNF $\alpha$ [17]. Even if the involvement of ICOS/ICOS-L pathway supported cancer progression and worsen prognosis in several cancer models, in colorectal cancer the expression of ICOS was associated with a good prognosis [13]. Although limited data is available on ICOS-L expression in colorectal cancer, ICOS is known to be mainly expressed on CD4 ${ }^{+} \mathrm{T}$ cells in patients' tumour tissues or peripheral blood cells [13]. Compared to ICOS-T cells, ICOS ${ }^{+} \mathrm{T}$ cells produce more INF $\gamma$ and less IL-4 and show up-regulated expression of transcriptional factor of Th1 cells (T-bet). This suggests that ICOS promotes Th1 effector response in patients with colon cancer. It is known that Th1 cells inhibit tumour cell invasion and metastasis by communicating with tumour-associated myeloid cells (i.e. tumour-associated macrophages and myeloid-derived suppressor cells) contributing to an improvement of survival. Studies showed a significant correlation between high ICOS expression and good prognosis, in relation to tumour size, Carcinoembryonic antigen (CEA) levels, tumor staging, lymphatic metastasis and distant metastasis (TNM classifications). Furthermore, ICOS expression was correlated with the expression of checkpoint inhibitors CTLA-4 and PD-1 on T cells, indicating that ICOS could also be a useful marker for a selection of anti-CTLA-4 or PD-1 therapy in colorectal cancer [13].

\subsection{ICOS/ICOS-L pathway in cancer immunotherapy}

The opposite effects of ICOS/ICOS-L pathway on the T-cell response may explain the lack of antitumor efficacy of ICOS-L blockade in mono-therapy [25]. Instead, studies blocking the ICOS/ICOS-L pathway in combination with other therapy showed different results. For example, therapy with GM-CSF-modified cancer cell vaccine combined with anti-ICOS monoclonal antibody induced more powerful anti-tumour immunity [18]. Indeed, Mo et al. demonstrated in murine models of prostate cancer how tumour-infiltrated T lymphocyte increased after treatment with GM-CSF-modified cancer cell vaccine and how combination therapy induced higher infiltration compared with vaccine alone. Furthermore, $\mathrm{ICOS}^{+} \mathrm{Foxp}^{+} \mathrm{T}$ cells infiltration into tumour tissues were higher after vaccine therapy, while the proportion of these cells decrease in combination treatmenr compared with control condition. Although the vaccine induced an efficient antitumor immunity inhibiting tumour growth, it also induced an increase of Tregs tumour infiltration that could challenge the effectiveness of this therapy. In this case, combination with ICOS blocking could deplete infiltrated Tregs with the possibility to enhance the vaccine-induced immunity [18].

Further supporting this evidence, treatment of pancreatic cancer targeting mesothelin (MSLN), a potential immunetherapeutic target, showed similar results. MSLN-virus-like particles (mMSLN-VLPs) immunization was able to break tolerance to intrinsic MSLN, resulting in reduced frequency of $\mathrm{CD}^{+}{ }^{+}$Foxp $3{ }^{+} \mathrm{ICOS}^{-}$Treg cells and stimulation of cytotoxic $\mathrm{CD}^{+} \mathrm{T}$ cells antitumor activity. However, because mMSLN-VLP induces IL-6 production, increasing ICOS-L expression on pDC-like cells and proliferation of immunosuppressive CD4 ${ }^{+} \mathrm{Foxp} 3^{+} \mathrm{ICOS}{ }^{+}$Treg cells, combination therapies with ICOS blocking remain necessary [19]. In an in vitro model of multiple myeloma, lenalidomide pre-treatment of MM cell 
lines reduced Treg generation and the Treg/TEff ratio, probably due to a reduced ICOS-L gene transcription. Combined treatment with lenalidomide and dexamethasone significantly reduced both Treg induction and the Treg/TEff cell ratio [21]. Additionally, depletion of Tregs in conjunction with ICOS agonist could remove the potential immunosuppressive effects of ICOS signalling and allow ICOS agonist to act solely in promoting activity of CD4 ${ }^{+}$Teff. In fact, ICOS is highly expressed in tumor Tregs, and thus a single ICOS agonist with strong Fc engagement may be sufficient to induce a simultaneous ADCC-mediated depletion of Tregs and agonist-based enhancement of anti-tumour responses [25].

Nevertheless, blocking of ICOS/ICOS-L system not always demonstrated to be good strategy. Results in in vivo models of prostate, melanoma [22] and bladder cancer [20], showed anti-CTLA-4 therapy leaded to an increase in the frequency of $\mathrm{CD}^{+}{ }^{+} \mathrm{ICOS}^{+}$and $\mathrm{CD}^{+} \mathrm{ICOS}^{+} \mathrm{T}$ cells producing IL-2 and IFN- $\gamma$. Here, ICOS seemed to play an important role in the activation/development of functional antitumor CD8 T cells [23]. In mouse syngeneic tumours, ICOS was found highly expressed by Tregs, but it was also found up-regulated across $\mathrm{CD} 8^{+}$and $\mathrm{CD} 4^{+}$effector populations, suggesting that ICOS expression was associated with opposite functions. According to this evidence, ICOS/ICOS-L pathway could be another therapeutic target and it may have implications in the development of novel combined cancer immunotherapy strategies $[22,23]$. Indeed, in murine models of prostate cancer, CTLA-4 blockade enhanced activation of tumour-reactive T cells with concomitant up-regulation of ICOS. In this context, IVAX (vaccine of ICOSL-positive tumour cells) triggered the ICOS pathway, leading to a higher density of Teff cells inside the tumour, as indicated by an increase in the Teff/Treg cell ratio [22]. Likewise, after anti-CTLA-4 treatment $\mathrm{ICOS}^{+} \mathrm{T}$ cells increased significantly in both the CD4 ${ }^{+}$and $\mathrm{CD} 8^{+}$ populations. In this case, anti-CTLA-4 therapy increased a population of Foxp3- cells and then stimulated an expansion of $\mathrm{ICOS}^{+} \mathrm{T}$ effector cells over Treg cells. This results suggest that $\mathrm{ICOS}^{+} \mathrm{T}$ cells may represent a population of $\mathrm{T}$ effector cells that play an important role in antitumor immune responses induced by anti-CTLA-4 therapy. Further, ICOS ${ }^{+} \mathrm{T}$ cells produced Th1 cytokine IFN- $\gamma$ and cytokine IL-2 necessary for effective antitumor responses, suggesting that ICOS $^{+} \mathrm{T}$ cells might play a functional role in improving the effectiveness of combinatorial immunotherapy. In a recent clinical trial, treatment of patients with bladder cancer were with a blocking anti-CTLA-4 mAb resulted in increased percentage of $\mathrm{CD}^{+}{ }^{+} \mathrm{T}$ cells in peripheral blood and tumour tissues, thus potential higher levels of ICOS. Upon re-stimulation, $\mathrm{ICOS}^{+} \mathrm{CD} 4^{+} \mathrm{T}$ cells produced greater levels of IFN- $\gamma$ compared to ICOS $\mathrm{CD}^{+} \mathrm{T}$ cells, suggesting that $\mathrm{T}$ cells with higher levels of ICOS, have elevated effector functions in antitumor activity, as seen in melanoma models [20].

\section{CONCLUSION}

The pathway ICOS/ICOS-L is involved in several processes in cancer, ranging from support of tumour growth but, at the same time, improvement of immune-stimulating therapy efficacy. Data suggest that inhibition of ICOS/ICOS-L system alone may not particularly effective in treatment of cancer because this pathway showed opposing effects in regulating T-cell response. The importance of ICOS/ICOS-L blockade was attributable to its role as adjuvant in combined cancer immunotherapy strategies. ICOS expression was positively correlated with the expression of other important immune checkpoints (CTLA-4 and PD-1 on T cells) in colon cancer patients, with antibodies anti-CTLA-4 and anti PD-1 currently being used for the treatment of several types of cancer. This suggest that ICOS could be a marker in therapy selection, with effectiveness depending on CTLA-4 or PD-1 expression correlated with ICOS expression. While increasing of Tregs in tumour microenvironment could reduce vaccine efficacy, a combination with anti-ICOS antibody might be a more effective therapy. This could increase immune response, as it would reduce the population of tumour infiltrated investigated in other cancers, taking into account that this pathway shows a dualistic behaviour. 


\section{Acknowledgements}

244 All authors have contributed to the writing and revision of the work. M.O. and N.B contributed equally to writing, M.B.M,

245 A.C and S.G. contributed to revision.

\section{Legends}

Figure 1. Alignment analysis of ICOS-L transcripts. NM_015259.5 (Vara, 3320 nt), NM_001283050.1 (Varb, 1188 nt), NM_001283051.1 (Varc, 2969 nt), NM_001283052.1 (Vard, 3168 nt). The analysis was performed by Clustal Omega software. * (Asterisk) indicates positions that have a single, fully conserved residue.

Figure 2. Sequence alignment analysis of ICOS-L protein variants. Vara (NP_056074.1, 302 aa), Varb (NP_001269979.1, 309 aa), Varc (NP_001269980.1, 185 aa), Vard (NP_001269981.1, 217 aa). * (asterisk) indicates positions which have a single, fully conserved residue. : (colon) indicates conservation between groups of strongly similar properties..(period) indicates conservation between groups of weakly similar properties. In Bold: trans-membrane domain, Clear: cytoplasmatic domain, Underline and clear: extracellular domain. Alignment analysis was performed by Clustar Omega software.

Figure 3. ICOS-ICOS-L pathways. ICOS-L is a member of B7 family expressed on professional antigen-presenting cells (APCs) and it binds ICOS receptor expressed on T cells. Activation of ICOS receptor induces the recruitment PI3K, a signalling molecule that leads to the activation of Akt, a kinase that promote cellular proliferation and survival. ICOS/ICOS-L pathway activation contributes to the production of cytokines, such as IL-5, IL-4, IL-13 and IL-10. It is suggested that ICOS/ICOS-L co-stimulatory signal is very important for memory and Thelper cell effector functions but, ICOS is seemingly capable of alternatively promoting or repressing T helper-1 responses by ICOS-dependent regulatory T cells (Tregs) induction.

Tab. 1 Differential role of ICOS-L/ICOS activation in different tumours. ICOS/ICOS-L pathway is activated in different types of cancer and it plays a central role in immune suppression. ICOS-L is present in tumour microenvironment because it is expressed by cancer cells or tumour-infiltrating plasmacytoid dendritic cells (pDCs). 


\section{REFERENCES}

[2] Wang, F.; Zhu, W.; Liu, T.; Sun, Z.; Ju, S.; Ju, S.; Yu, G.; Xie, W.; Deng, Z.; Lu, B.; Zhang, X. The expression analysis of ICOS-L on activated T cells and immature dendritic cells as well as malignant B cells and Grave's-diseasederived thyroid tissues by two novel mAbs against human ICOS-L. Tissue Antigens, 2007, 69, 62-72.

[3] Wikenheiser, D.J.; Stumhofer, J.S. ICOS Co-Stimulation: Friend or Foe? Front Immunol, 2016, 7, 304.

[4] Yoshinaga, S.K.; Zhang, M.; Pistillo, J.; Horan, T.; Khare, S.D.; Miner, K.; Sonnenberg, M.; Boone, T.; Brankow, D.; new human B7-related protein: B7RP-1 is the ligand to the co-stimulatory protein ICOS. Int Immunol, 2000, 12(10), $1439-1447$.

[5] Aicher, A.; Hayden-Ledbetter, M.; Brady, W.A.; Pezzutto, A.; Richter, G.; Magaletti, D.; Buckwalter, S.; Ledbetter, J.A.; Clark, E.A. Characterization of human inducible costimulator ligand expression and function. $J$ Immunol, 2000, 164(9), 4689-4696.

[6] Nagase, H.; Takeoka, T.; Urakawa, S.; Morimoto-Okazawa, A.; Kawashima, A.; Iwahori, K.; Takiguchi, S.; Nishikawa, H.; Sato, E.; Sakaguchi, S.; Mori, M.; Doki, Y.; Wada, H. ICOS(+) Foxp3(+) TILs in gastric cancer are prognostic markers and effector regulatory T cells associated with Helicobacter pylori. Int J Cancer, 2017, 140, 686-695.

[7] Martin-Orozco, N.; Li, Y.; Wang, Y.; Liu, S.; Hwu, P.; Liu, Y.J.; Dong, C.; Radvanyi, L. Melanoma cells express 297

[9] Yamashita, T.; Tamura, H.; Satoh, C.; Shinya, E.; Takahashi, H.; Chen, L.; Kondo, A.; Tsuji, T.; Dan, K.; Ogata, K. Functional B7.2 and B7-H2 molecules on myeloma cells are associated with a growth advantage. Clin Cancer Res, 2009, 15(3), 770-777.

[10] Faget, J.; Bendriss-Vermare, N.; Gobert, M.; Durand, I.; Olive, D.; Biota, C.; Bachelot, T.; Treilleux, I.; Goddard308 dendritic cells support breast cancer progression by promoting the accumulation of immunosuppressive CD4+ T cells. Cancer Res, 2012, 72(23), 6130-6141. 
[11] Conrad, C.; Gregorio, J.; Wang, Y.H.; Ito, T.; Meller, S.; Hanabuchi, S.; Anderson, S.; Atkinson, N.; Ramirez, P.T.; Liu, Y.J.; Freedman, R.; Gilliet, M. Plasmacytoid dendritic cells promote immunosuppression in ovarian cancer via ICOS co-stimulation of Foxp3(+) T-regulatory cells. Cancer Res, 2012, 72(20), 5240-5249.

[12] Pedroza-Gonzalez, A.; Zhou, G.; Vargas-Mendez, E.; Boor, P.P.; Mancham, S.; Verhoef, C.; Polak, W.G.; Grünhagen, D.; Pan, Q.; Janssen, H.; Garcia-Romo, G.S.; Biermann, K.; Tjwa, E.T.; IJzermans, J.N.; Kwekkeboom, J.; Sprengers, D. Tumor-infiltrating plasmacytoid dendritic cells promote immunosuppression by $\operatorname{Tr} 1$ cells in human liver tumors. Oncoimmunology, 2015, 4(6), e1008355.

[13] Zhang, Y.; Luo, Y.; Qin, S.L.; Mu, Y.F.; Qi, Y.; Yu, M.H.; Zhong, M. The clinical impact of ICOS signal in colorectal cancer patients. Oncoimmunology, 2016, 5(5), e1141857.

[14] Greaves, P.; Clear, A.; Owen, A.; Iqbal, S.; Lee, A.; Matthews, J.; Wilson, A.; Calaminici, M.; Gribben, J.G. Defining characteristics of classical Hodgkin lymphoma microenvironment T-helper cells. Blood, 2013, 122(16), 2856-2863.

[15] Tamura, H.; Dan, K.; Tamada, K.; Nakamura, K.; Shioi, Y.; Hyodo, H.; Wang, S.D.; Dong, H.; Chen, L.; Ogata, K. Expression of functional B7-H2 and B7.2 costimulatory molecules and their prognostic implications in de novo acute myeloid leukemia. Clin Cancer Res, 2005, 11(16), 5708-5717.

[16] Schreiner, B.; Wischhusen, J.; Mitsdoerffer, M.; Schneider, D.; Bornemann, A.; Melms, A.; Tolosa, E.; Weller, M.; Wiendl, H. Expression of the B7-related molecule ICOSL by human glioma cells in vitro and in vivo. Glia, 2003, 44(3), 296-301.

[17] Simon-Keller, K.; Paschen, A.; Hombach, A.A.; Ströbel, P.; Coindre, J.M.; Eichmüller, S.B.; Vincent, A.; Gattenlöhner, S.; Hoppe, F.; Leuschner, I.; Stegmaier, S.; Koscielniak, E.; Leverkus, M.; Altieri, D.C.; Abken, H.; Marx, A. Survivin blockade sensitizes rhabdomyosarcoma cells for lysis by fetal acetylcholine receptor-redirected T cells. Am J Pathol, 2013, 182(6), 2121-2131.

[18] Mo, L.; Chen, Q.; Zhang, X.; Shi, X.; Wei, L.; Zheng, D.; Li, H.; Gao, J.; Li, J.; Hu, Z. Depletion of regulatory T cells by anti-ICOS antibody enhances anti-tumor immunity of tumor cell vaccine in prostate cancer. Vaccine, 2017, 35(43), 5932-5938.

[19] Zhang, S.; Yong, L.K.; Li, D.; Cubas, R.; Chen, C.; Yao, Q. Mesothelin virus-like particle immunization controls pancreatic cancer growth through CD8+ $\mathrm{T}$ cell induction and reduction in the frequency of CD4+ foxp3+ ICOSregulatory T cells. PLoS One, 2013, 8(7), e68303.

[20] Chen, H.; Liakou, C.I.; Kamat, A.; Pettaway, C.; Ward, J.F.; Tang, D.N.; Sun, J.; Jungbluth, A.A.; Troncoso, P.; Logothetis, C.; Sharma, P. Anti-CTLA-4 therapy results in higher CD4+ICOShi T cell frequency and IFN-gamma levels in both nonmalignant and malignant prostate tissues. Proc Natl Acad Sci U S A, 2009, 106(8), 2729-2734. 
351 [21] Scott, G.B.; Carter, C.; Parrish, C.; Wood, P.M.; Cook, G. Downregulation of myeloma-induced ICOS-L and regulatory $\mathrm{T}$ cell generation by lenalidomide and dexamethasone therapy. Cell Immunol, 2015, 297(1), 1-9.

\section{3}

354 355 356 357 358 359 360 361 362 363 364 365 366 367
[22] Fan, X.; Quezada, S.A.; Sepulveda, M.A.; Sharma, P.; Allison, J.P. Engagement of the ICOS pathway markedly enhances efficacy of CTLA-4 blockade in cancer immunotherapy. $J$ Exp Med, 2014, 211(4), 715-725.

[23] Fu, T.; He, Q.; Sharma, P. The ICOS/ICOSL pathway is required for optimal antitumor responses mediated by antiCTLA-4 therapy. Cancer Res, 2011, 71(16), 5445-5454.

[24] Ito, T.; Yang, M.; Wang, Y.H.; Lande, R.; Gregorio, J.; Perng, O.A.; Qin, X.F.; Liu, Y.J.; Gilliet, M. Plasmacytoid dendritic cells prime IL-10-producing T regulatory cells by inducible costimulator ligand. J Exp Med, 2007, 204(1), 105115 .

[25] Metzger, T.C.; Long, H.; Potluri, S.; Pertel, T.; Bailey-Bucktrout, S.L.; Lin, J.C.; Fu, T.; Sharma, P.; Allison, J.P.; Feldman, R.M. ICOS Promotes the Function of CD4+ Effector T Cells during Anti-OX40-Mediated Tumor Rejection. Cancer Res, 2016, 76(13), 3684-3689. 